\title{
Some Basic Properties of Packing and Covering Constants
}

\author{
H. Groemer* \\ Department of Mathematics, The University of Arizona, Tucson, AZ 85710
}

\begin{abstract}
This article concerns packings and coverings that are formed by the application of rigid motions to the members of a given collection $\mathbf{K}$ of convex bodies. There are two possibilities to construct such packings and coverings: One may permit that the convex bodies from $\mathbf{K}$ are used repeatedly, or one may require that these bodies should be used at most once. In each case one can define the packing and covering constants of $\mathbf{K}$ as, respectively, the least upper bound and the greatest lower bound of the densities of all such packings and coverings. Three theorems are proved. First it is shown that there exist always packings and coverings whose densities are equal to the corresponding packing and covering constants. Then, a quantitative continuity theorem is proved which shows in particular that the packing and covering constants depend, in a certain sense, continuously on $\mathbf{K}$. Finally, a kind of a transference theorem is proved, which enables one to evaluate the packing and covering constants when no repetitions are allowed from the case when repetitions are permitted. Furthermore, various consequences of these theorems are discussed.
\end{abstract}

\section{Introduction}

Let $E^{n}$ denote $n$-dimensional euclidean space, and let $\mathbf{K}=\left\{K_{\nu}\right\}$ be an indexed collection of convex bodies in $E^{n}$. Thus, each $K_{\nu}$ is assumed to be a compact convex subset of $E^{n}$ with nonempty interior, and $K_{v}=K_{\mu}$ holds if and only if $\nu=\mu$. Let $I$ denote the group of all isometries and $T$ the group of all translations of $E^{n}$, and let $G$ be a subgroup of $I$ that contains $T$. We view $\mathbf{K}$ as a "supply" of sets and consider packings and coverings that are constructed by the application of rigid motions from $G$ to the convex bodies of $K$. In the traditional

* Supported by National Science Foundation Research Grant DMS 8300825. 
theory of packings and coverings (cf. L. Fejes Tóth [3] or Rogers [9]) one usually starts with a very small set $K$ (in most cases consisting of one element only) but permits repeated use of the elements of this set. The following definition corresponds to this type of packings and coverings.

Definition 1. Let $\mathbf{K}=\left\{K_{v}\right\}_{v \in N}$ be an indexed collection of convex bodies in $E^{\prime \prime}$. A class $\mathbf{C}$ of convex bodies will be called a $\mathbf{K}^{r}$-packing in $E^{n}$ (with respect to $G)$ if the following conditions are satisfied:

(a) For each $\nu \in N$ there is a (possibly empty) subset $G_{\nu}$ of $G$ such that $\mathbf{C}=U_{\nu \in N}\left\{g \mathrm{~K}_{\nu}: g \in G_{\nu}\right\}$.

(b) Every ball in $E^{n}$ meets only finitely many sets of $\mathrm{C}$.

(c) The interiors of any two sets from $\mathbf{C}$ are disjoint.

Similarly, $\mathbf{C}$ is called a $\mathbf{K}^{r}$-covering of $E^{n}$ (with respect to $G$ ) if (a) and (b) are satisfied and

(d) $E^{n} \subset \cup C_{i} \quad\left(C_{i} \in \mathrm{C}\right)$.

In this definition the superscript $r$ indicates that the sets from $\mathbf{K}$ may be used repeatedly, i.e., some of the sets $G_{v}$ may have more than one element. More recently another possibility to generate packings and coverings has received some attention (see the survey [8]). One may start with a large set $\mathbf{K}$ (usually an infinite sequence) and use each $K_{\nu} \in \mathbf{K}$ at most once. The following definition describes this situation.

Definition 2. Let $\mathbf{K}$ be a set as in Definition 1. A class $\mathbf{C}$ of convex bodies is called a K-packing or K-covering if, respectively, (a), (b), and (c), or (a), (b), and (d) hold, and, in addition, the sets $G_{v}$ can be selected so that each of them has at most one element.

In investigations dealing with this kind of packings and coverings it is often assumed that each $G_{v}$ should contain exactly one element, i.e., each set of $\mathbf{K}$ should actually be used. However, this additional requirement is immaterial. Indeed, if $\mathbf{K}$ is countable and not all sets are used, it is easy to modify the packings and coverings so that all sets are used and the densities remain the same; and if $\mathbf{K}$ is not countable, it is impossible to use all its members (due to (b)).

If $\mathbf{C}$ is a $\mathbf{K}$-packing, $\mathbf{K}^{r}$-packing, $\mathbf{K}$-covering, or $\mathbf{K}^{r}$-covering (with respect to a given group $G$ ), then its density is defined by

$$
\delta(\mathbf{C})=\lim _{t \rightarrow \infty} \frac{1}{v\left(B_{t}\right)} \sum_{C_{t} \in \mathbf{C}} v\left(B_{t} \cap C_{i}\right),
$$

where $B_{t}$ denotes the ball in $E^{n}$ of radius $t$ with center at the origin, and $v$ denotes volume in $E^{n}$. Since $\delta(C)$ does not always exist, it is often useful to introduce the upper density and the lower density of $\mathbf{C}$. These are defined, 
respectively, by

$$
\bar{\delta}(\mathbf{C})=\varlimsup_{t \rightarrow \infty} \frac{1}{v\left(B_{t}\right)} \sum_{C_{t} \in \mathbf{C}} v\left(B_{t} \cap C_{i}\right)
$$

and

$$
\underline{\delta}(\mathbf{C})=\lim _{t \rightarrow \infty} \frac{1}{v\left(B_{t}\right)} \sum_{C_{i} \in \mathbf{C}} v\left(B_{t} \cap C_{t}\right) .
$$

If the set of the diameters of all $C_{i}$ is bounded, one can prove that these densities do not depend on the choice of the origin of $E^{n}$ and that the sums $\sum v\left(B_{t} \cap C_{1}\right)$ could be replaced by $\sum v\left(C_{1}\right)$ taken over all $C_{i}$ with $C_{t} \subset B_{t}$ or over all $C_{t}$ with $B_{t} \cap C_{t} \neq \varnothing$ (see [5], Satz 4). The packing and covering constants of $\mathbf{K}$ (with respect to $G$ ) can now be defined by

$$
\begin{aligned}
& d_{p}(\mathbf{K}, G)=\sup \{\bar{\delta}(\mathbf{C}): \mathbf{C} \text { is a } \mathbf{K} \text {-packing with respect to } G\}, \\
& d_{p}^{r}(\mathbf{K}, G)=\sup \left\{\bar{\delta}(\mathbf{C}): \mathbf{C} \text { is a } \mathbf{K}^{r} \text {-packing with respect to } G\right\}, \\
& d_{c}(\mathbf{K}, G)=\inf \{\underline{\delta}(\mathbf{C}): \mathbf{C} \text { is a } \mathbf{K} \text {-covering with respect to } G\}, \\
& d_{c}^{r}(\mathbf{K}, G)=\inf \left\{\underline{\delta}(\mathbf{C}): \mathbf{C} \text { is a } \mathbf{K}^{r} \text {-covering with respect to } G\right\} .
\end{aligned}
$$

We note that the definition of $d_{c}(\mathbf{K}, G)$ is only of interest if there is at least one $\mathbf{K}$-covering with respect to $G$. This is not always the case, even if $\mathbf{K}$ is infinite.

The above definitions reflect the fact that one is primarily interested in packings of high density and coverings of low density. In fact, a large part of the theory of packings and coverings is, directly or indirectly, concerned with the evaluation or estimation of packing and covering constants.

If the group $G$ is determined by the context in which it appears, or if $G$ is immaterial for a particular statement, we simplify the notation by writing $d_{p}(\mathbf{C})$, $d_{p}^{r}(\mathbf{C}), d_{c}(\mathbf{C})$, and $d_{c}^{r}(\mathbf{C})$ instead of $d_{p}(\mathbf{C}, G), d_{p}^{r}(\mathbf{C}, G), d_{c}(\mathbf{C}, G)$, and $d_{c}^{r}(\mathbf{C}, G)$.

In this article three rather basic theorems on packing and covering constants will be established. First we prove that under very general assumptions there are always packings and coverings whose densities equal, respectively, $d_{p}(\mathbf{K}), d_{p}^{r}(\mathbf{K})$, $d_{c}(\mathbf{K})$, and $d_{c}^{r}(\mathbf{K})$. In the case of $d_{p}^{r}(\mathbf{K})$ and $d_{c}^{r}(\mathbf{K})$ such theorems have already been proved in [5], but if no repetitions are allowed, some additional considerations are necessary. Then we show that the packing and covering constants depend continuously on $\mathbf{K}$. In fact, we give explicit estimates to this effect. As a consequence of this theorem we obtain a limit theorem concerning tilings of $E^{n}$. Finally we show that under suitable assumptions it is possible to evaluate $d_{p}(\mathbf{K})$ and $d_{c}(\mathbf{K})$ if $d_{p}^{r}\left(\mathbf{K}^{\prime}\right)$ and $d_{c}^{r}\left(\mathbf{K}^{\prime}\right)$ are given, where $\mathbf{K}^{\prime}$ consists of the appropriately defined limit elements of $\mathbf{K}$. Since $d_{p}^{r}$ and $d_{c}^{r}$ are usually much easier to deal with than $d_{p}$ and $d_{c}$, this theorem has several remarkable consequences.

An indexed infinite collection of convex bodies in $E^{n}$ will often be referred to as a supply set (since it supplies the convex bodies for our packings and coverings). 


\section{Theorems and Corollaries}

Existence Theorem. If $\mathbf{K}=\left\{K_{v}\right\}$ is a supply set in $E^{n}$ such that the set of the diameters of the convex bodies $K_{\nu}$ is bounded, then there exist a $\mathbf{K}$-packing $\mathbf{P}, a$ $\mathbf{K}^{r}$-packing $\mathbf{P}^{*}$, a $\mathbf{K}$-covering $\mathbf{C}$ (provided that there is at least one $\mathbf{K}$-covering), and a $\mathbf{K}^{r}$-covering $\mathbf{C}^{*}$ such that the densities of $\mathbf{P}, \mathbf{P}^{*}, \mathbf{C}$, and $\mathbf{C}^{*}$ exist and $\delta(\mathbf{P})=d_{p}(\mathbf{K}), \delta\left(\mathbf{P}^{*}\right)=d_{p}^{r}(\mathbf{K}), \delta(\mathbf{C})=d_{c}(\mathbf{K})$, and $\delta\left(\mathbf{C}^{*}\right)=d_{c}^{r}(\mathbf{K})$.

These statements are true with respect to any given group $G$ of isometries of $E^{n}$ that contains the translation group $T$. The packing and covering constants are, of course, taken with respect to this $G$.

The Existence Theorem shows that in the definition of the packing and covering constants one may replace $\bar{\delta}$ and $\delta$ by $\delta$, and restrict the attention to packings and coverings whose densities exist.

To formulate our second theorem we let $h(M, N)$ denote the Hausdorff distance between two convex bodies $M$ and $N$. Furthermore, if $\mathbf{K}=\left\{K_{\nu}\right\}$ and $\mathbf{L}=\left\{L_{\nu}\right\}$ are two supply sets with equal index set, say $J$, we extend the definition of $h$ by setting

$$
h(\mathbf{K}, \mathbf{L})=\sup _{\nu \in J} h\left(K_{\nu}, L_{\nu}\right) .
$$

Continuity Theorem. Let $\mathbf{K}=\left\{K_{\nu}\right\}$ and $\mathbf{L}=\left\{L_{\nu}\right\}$ be two supply sets in $E^{n}$ with equal index set. Assume that there are two constants $a, b$ such that $0<a \leq b<\infty$ and every $K_{\nu}$ and $L_{\nu}$ has inradius at least $a$ and circumradius at most $b$. Furthermore, let $\varepsilon$ be a number with $0<\varepsilon<a$. Then, the condition $h(\mathbf{K}, \mathbf{L}) \leq \varepsilon$ implies

$$
\begin{aligned}
& \left|d_{p}(\mathbf{K})-d_{p}(\mathbf{L})\right| \leq \frac{\varepsilon}{a}\left(4^{n}-1\right), \\
& \left|d_{p}^{r}(\mathbf{K})-d_{p}^{r}(\mathbf{L})\right| \leq \frac{\varepsilon}{a}\left(4^{n}-1\right),
\end{aligned}
$$

and

$$
\begin{aligned}
& \left|d_{c}(\mathbf{K})-d_{c}(\mathbf{L})\right| \leq \frac{\varepsilon}{a}\left(4^{n}-1\right) \max \left\{d_{c}(\mathbf{K}), d_{c}(\mathbf{L})\right\}, \\
& \left|d_{c}^{r}(\mathbf{K})-d_{c}^{r}(\mathbf{L})\right| \leq \frac{\varepsilon}{a}\left(4^{n}-1\right) \max \left\{d_{c}^{r}(\mathbf{K}), d_{C}^{r}(\mathbf{L})\right\} .
\end{aligned}
$$

We note that $\max \left\{d_{c}^{r}(\mathbf{K}), d_{c}^{r}(\mathbf{L})\right\} \leq \max \left\{d_{c}(\mathbf{K}), d_{c}(\mathbf{L})\right\} \leq(b / a)^{n} \beta_{n}$, where $\beta_{n}$ is the covering constant for congruent balls.

As an application of this theorem we consider tilings of $E^{n}$. By a $\mathbf{K}^{r}$-tiling of $E^{n}$ (with respect to $G$ ) we mean a $\mathbf{K}^{r}$-packing that is at the same time a $\mathbf{K}^{r}$-covering (both with respect to $G$ ). If there exists such a tiling, we call $\mathbf{K}$ a tiling set (with respect to $G$ ). Let now $\mathbf{K}_{i}$ be a sequence of tiling sets that converges to some supply set $\mathbf{K}$ in the sense that $\lim _{i \rightarrow \infty} h\left(\mathbf{K}_{i}, \mathbf{K}\right)=0$, and assume that there are constants $a, b$ such that $0<a \leq b<\infty$ and every $K_{\nu} \in \mathbf{K}$ has inradius at least $a$ and circumradius at most $b$. Since $d_{p}^{r}\left(\mathbf{K}_{i}\right)=1$ (for all $i$ ) it follows from the Continuity Theorem that $d_{p}^{r}(\mathbf{K})=1$. If $G$ is a closed subgroup of $I$, then the 
closure of $\mathbf{K}$, say $\overline{\mathbf{K}}$, is also a tiling set. If $K \subset B_{b}$ for all $K \in \overline{\mathbf{K}}$ and $G=T$ a proof of this fact is given in [4]. The general situation can be reduced to this case by considering instead of $\overrightarrow{\mathbf{K}}$ the set $\left\{\gamma K_{\nu}+p: K_{\nu} \in \overline{\mathbf{K}}, \gamma \in G, p \in E^{n}, K_{\nu}+p \subset B_{b}\right\}$. Thus, we obtain the following corollary.

Corollary 1. For $i=1,2, \ldots$ let $\mathbf{K}_{i}$ be a tiling set in $\mathbf{E}^{n}$ (with respect to a given closed group G), such that $\lim _{t \rightarrow \infty} h\left(\mathbf{K}_{t}, \mathbf{K}\right)=0$, where $\mathbf{K}$ is a supply set with the property that the set of the inradii of the members of $\mathbf{K}$ has a positive lower bound and the set of circumradii a finite upper bound. Then, $\overline{\mathbf{K}}$ is again a tiling set (with respect to G). In particular, if for $i=1,2, \ldots$ each $\mathbf{K}_{i}=\left\{K_{t}^{1}, \ldots, K_{t}^{m}\right\}$ consists of $m$ convex bodies and if for $j=1,2, \ldots, m$ each of the sequences $K_{1}, K_{2}^{j}, \ldots$ converges to a convex body $K^{j}$, then $\left\{K^{1}, \ldots, K^{m}\right\}$ is again a tiling set (always with respect to the same closed group).

If $\mathbf{K}$ is a given supply set we let $\mathbf{K}^{\prime}$ denote the set of all limit elements of $\mathbf{K}$. Thus, $\overline{\mathbf{K}}=\mathbf{K} \cup \mathbf{K}^{\prime}$ and a set is in $\mathbf{K}^{\prime}$ exactly if it is the limit of a sequence of distinct members of $\mathbf{K}$. (Since $\mathbf{K}$ is always assumed to be an indexed collection of convex bodies, "distinct" means that the pertinent subscripts are distinct.) $\mathbf{K}$ will be said to be bounded if the set of the inradii of the members of $\mathbf{K}$ has a positive lower bound and there is a ball that contains all members of $\mathbf{K}$. Using these definitions we can formulate our third theorem.

Transference Theorem. Let $\mathbf{K}$ be a bounded supply set in $E^{n}$, and let $\mathbf{K}^{\prime}$ be the set of limit elements of $\mathbf{K}$. Then,

$$
d_{p}(\mathbf{K})=d_{p}^{r}\left(\mathbf{K}^{\prime}\right)
$$

and

$$
d_{c}(\mathbf{K})=d_{c}^{r}\left(\mathbf{K}^{\prime}\right)
$$

(with respect to any group of isometries containing T).

The following corollary is an immediate consequence of this theorem.

Corollary 2. If the bounded supply set $\mathbf{K}$ has only one limit element, say $\mathbf{K}$, then $d_{p}(\mathbf{K})=d_{p}^{r}(\{K\})$ and $d_{c}(\mathbf{K})=d_{c}^{r}(\{K\})$.

Hence, when dealing with a bounded supply set which has only one limit element one achieves essentially the same packing or covering effect if only one set (and the same group) is used. But even if $\mathbf{K}$ has more than one limit element, it is still possible to obtain some information on $d_{p}(\mathbf{K})$ and $d_{c}(\mathbf{K})$, provided that estimates of the packing or covering constants for copies of single convex bodies are available. The following corollary is useful for this purpose.

Corollary 3. Let $k_{n}(G)$ be a constant such that for every convex body $M$ either $d_{p}^{r}(\{M\}, G) \geq k_{n}(G)$ or $d_{c}^{r}(\{M\}, G) \leq k_{n}(G)$. Then, for every bounded supply set $\mathbf{K}$ the corresponding inequality $d_{p}(\mathbf{K}, G) \geq k_{n}(G)$ or $d_{c}(\mathbf{K}, G) \leq k_{n}(G)$ holds.

For explicit values of $k_{n}(G)$ that can be used in this corollary see the pertinent sections in Rogers [9], L. Fejes Tóth [3], and G. Fejes Tóth [2]. As an 
example consider packings and coverings in $E^{2}$ with respect to the translation group $T$. In this case one may take $k_{n}(T)$ to be $\frac{2}{3}$ in the case of packings and $\frac{3}{2}$ for coverings (see [3], p. 100). Hence, for every bounded supply set $\mathbf{K}$ in $E^{2}$ we have

$$
d_{p}(\mathbf{K}, T) \geq \frac{2}{3}
$$

and

$$
d_{c}(\mathbf{K}, T) \leq \frac{3}{2} \text {. }
$$

These inequalities are best possible; equality holds when $\mathbf{K}$ consists of translates of a triangle. Without the assumption of bondedness of the supply set, inequalities of this kind (with respect to $T$ ) are rather difficult to prove and practically unknown when $n>2$ (see Groemer $[7,8]$ ).

If the Transference Theorem is applied to ellipsoids, it yields immediately the following result.

Corollary 4. If $K$ is a bounded supply set in $E^{n}$ consisting of (solid) ellipsoids, then $d_{p}(\mathbf{K}, T) \geq \alpha_{n}$ and $d_{c}(\mathbf{K}, T) \leq \beta_{n}$, where $\alpha_{n}$ and $\beta_{n}$ are, respectively, the packing and covering constants for congruent balls.

If $\mathbf{K}$ is a supply set consisting of (not necessarily congruent) simplexes, it will, in general, not be possible to find $\mathbf{K}$-packings or $\mathbf{K}$-coverings with respect to $I$ of density 1 . However, the following corollary shows that after an affine transformation of the members of $\mathbf{K}$ (the same transformation for all members) such packings and coverings are possible. Since $\mathbf{K}^{\prime}$ will also consist of simplexes, this is a consequence of the Transference Theorem and the fact that there are simplexes that permit tilings of $E^{n}$ (cf. Danzer [1]).

Corollary 5. If $\mathbf{K}$ is a bounded supply set of simplexes in $E^{n}$, there is an affine transformation $\sigma$ of $E^{n}$ such that $d_{p}(\sigma \mathbf{K}, I)=1$ and $d_{c}(\sigma \mathbf{K}, I)=1$, where $\sigma \mathbf{K}=$ $\left\{\sigma K_{v}: K_{\nu} \in \mathbf{K}\right\}$ and I denotes the group of isometries of $E^{n}$.

Finally, we consider G-tiles, i.e., convex bodies $K$ such that $\{K\}$ is a tiling set with respect to the group $G(T \subset G \subset I)$. If $\mathbf{K}=\left\{K_{\nu}\right\}$ is a collection of such tiles, for example a set of (not necessarily congruent) parallelotopes, it is an interesting question whether some of these $K_{\nu}$ can be rearranged (by the application of isometries from $G$ ) so that they form a packing or covering of density 1 . If $\mathbf{K}$ is bounded the following corollary gives an affirmative answer to this question; it follows immediately from the Transference Theorem and Corollary 1.

Corollary 6. Let $\mathbf{K}$ be a bounded supply set with the property that each member of $\mathbf{K}$ is a $G$-tile. Then, $d_{p}(\mathbf{K}, G)=1$ and $d_{c}(\mathbf{K}, G)=1$. In particular, if $\mathbf{K}$ is $a$ countable bounded supply set of parallelotopes, then one can rearrange these parallelotopes by the application of translations so that they form a packing of density 1 or a covering of density 1.

The very last statement of this corollary is also proved in [6]. In the case $n=2$, or when all isometries are permitted one can prove an analogous result for 
packings and coverings by orthogonal parallelotopes without the assumption that the set of the inradii of these parallelotopes have a positive lower bound (see Groemer [7]).

\section{Proofs of the Theorems}

Proof of the Existence Theorem. First we consider K-packings in $E^{n}$. Because of the condition on the diameters of the members of $\mathbf{K}$ there is a number $b$ such that the circumradius of every $K_{v} \in \mathbf{K}$ is at most $b$. We let $W_{s}$ denote the cube $\left\{\left(x_{1}, \ldots x_{n}\right):-\frac{1}{2} s \leq x_{i} \leq \frac{1}{2} s\right\}$, and $\Gamma$ the set of all points of $E^{n}$ with integral coordinates. Using standard notational conventions we can state that $W_{s}+s \Gamma$ is a tiling of $E^{n}$. For $m=1,2, \ldots$ we set

$$
\begin{aligned}
& s_{m}=2^{m}, \\
& t_{m}=2^{\left(m^{2}\right)},
\end{aligned}
$$

and consider the sequence of balls $B_{t_{0}} \subset B_{t_{1}} \subset B_{t_{2}} \subset \ldots$. Let $Q_{1}^{m}, \ldots, Q_{k_{m}}^{m}$ be those cubes of $W_{s_{m}}+s_{m} \Gamma$ that are contained in $B_{t_{m}}$ int $B_{t_{m-1}}$. We show that for each $Q_{i}^{m}$ there is a finite (possibly empty) collection $\mathbf{S}_{i}^{m-1}=\left\{L_{l, 1}^{m}, L_{i, 2}^{m}, \ldots\right\}$ of convex bodies so that the following conditions are satisfied:

(i) Each $L_{i, j}^{m}$ is of the form $g_{v} K_{\nu}$ with $g_{v} \in G, K_{v} \in \mathbf{K}$;

(ii) $L_{i, j}^{m} \subset Q_{i}^{m}$ for all $j$;

(iii) (int $L_{i, j}^{m}$ ) $\cap$ (int $L_{i, l}^{m}$ ) $=\varnothing$ unless $j=l$;

(iv) all $L_{i, j}^{m}$ originate from different members of $\mathbf{K}$;

(v) $\left(1 / s_{m}^{n}\right) \sum_{j} v\left(L_{i, j}^{m}\right) \geq h_{m}$, where $h_{m}=\left(\left(s_{m}-4 b\right) / s_{m}\right)^{n}\left(d_{p}(K)-1 / m\right)$ (or 0 if one of the two factors is negative).

If $m=1$, then $h_{m}=0$ and we take $\mathbf{S}_{i}^{m}=\varnothing$ (as we do in all other cases when $h_{m}=0$ ). Assuming that all the collections $\mathbf{S}_{i}^{m-1}$ have already been found, we determine for $i=1, \ldots, k_{m}$ the collection $\mathbf{S}_{i}^{m}$ by the following procedure.

There exists a K-packing, say $\left\{K_{q}^{m}\right\}$, of upper density at least $d_{p}(\mathbf{K})-$ $(1 /(m+1))$. Since the removal of finitely many sets from $\left\{K_{q}^{m}\right\}$ does not change the upper density, we may assume that $\left\{K_{q}^{m}\right\}$ does not contain any element that has already been used previously to define one of the sets associated with $Q_{r}^{k}$, where $k \leq m-1$, or $k=m$ and $1 \leq i-1$. There must exist a cube $W_{s_{m}-4 b}+g$ from the tiling $W_{s_{m}-4 b}+\left(s_{m}-4 b\right) \Gamma$ such that

$$
\frac{1}{\left(s_{m}-4 b\right)^{n}} \sum_{q} v\left(K_{q}^{m} \cap\left(W_{s_{m}-4 b}+g\right)\right)>d_{p}(\mathbf{K})-\frac{1}{m}
$$

(otherwise the upper density of $\left\{K_{q}^{m}\right\}$ would be at most $d_{p}(\mathbf{K})-1 / m<d_{p}(\mathbf{K})-$ $1 /(m+1)$, in contradiction to our assumption). Since the sets $K_{q}^{m}$ with $K_{q}^{m} \cap$ $\left(W_{s_{m}-4 h}+g\right) \neq \varnothing$ are contained in $W_{s_{m}}+g$, we have

$$
\frac{1}{\left(s_{m}-4 b\right)^{n}} \sum_{K_{q}^{m} \subset W_{s_{m}}+g} v\left(K_{q}^{m} \cap\left(W_{s_{m}}+g\right)\right)>d_{p}(\mathbf{K})-\frac{1}{m} .
$$


If $Q_{i}^{m}=W_{s_{m}}+g_{i}$, we take as the sets $L_{i, j}^{m}$ the translates $K_{q}^{m}+g_{1}-g$ of all $K_{q}^{m}$ with $K_{q}^{m} \subset W_{s_{m}}+g$. Then, (v) is obviously true. Since it is also clear that for this choice of the sets $L_{t, j}^{m}$ conditions (i), (ii), (iii), and (iv) are satisfied, it follows that the class of all $L_{i, j}^{m}$, forms a K-packing, say $\mathbf{L}$. Setting $\left\{L_{t, j}^{m}\right\}=\left\{M_{k}\right\}$ we prove now that

$$
\lim _{s \rightarrow \infty} \frac{1}{v\left(B_{s}\right)} \sum_{k} v\left(M_{k} \cap B_{s}\right) \geq d_{p}(\mathbf{K}) .
$$

If $s>2$ is given, let $m$ be determined by $t_{m}<s \leq t_{m+1}$. Collecting for each cube $Q_{i}^{m}$ the sets $M_{k}$ which are contained in it, and using (v) and the fact that $v\left(Q_{i}^{m}\right)=s_{m}^{n}$ we find

$$
\begin{aligned}
& \frac{1}{v\left(B_{s}\right)} \sum_{k} v\left(M_{k} \cap B_{s}\right)= \frac{1}{v\left(B_{s}\right)} \sum_{k} v\left(M_{k} \cap B_{t_{m-1}}\right) \\
&+\frac{1}{v\left(B_{s}\right)} \sum_{k} v\left(M _ { k } \cap \left(B_{\left.\left.t_{m} \backslash B_{t_{m-1}}\right)\right)}\right.\right. \\
&+\frac{1}{v\left(B_{s}\right)} \sum_{k} v\left(M_{k} \cap\left(B_{s} \backslash B_{t_{m}}\right)\right) \\
& \geq \frac{h_{m}}{v\left(B_{s}\right)} \sum_{Q_{t}^{m} \subset B_{t_{m} \backslash B_{t_{m-1}}} v\left(Q_{i}^{m}\right)} \\
&+\frac{h_{m+1}}{v\left(B_{s}\right)} \sum_{Q_{t}^{m+1} \subset B_{\mathfrak{s}} \backslash B_{t_{m}}} v\left(Q_{i}^{m+1}\right) \\
& \geq \frac{h_{m}}{v\left(B_{s}\right)}\left(v\left(B_{t_{m}-\rho_{m}}\right)\right. \\
&\left.\quad-v\left(B_{t_{m-1}+\rho_{m}}\right)+v\left(B_{s-\rho_{m}}\right)-v\left(B_{t_{m}+\rho_{m}}\right)\right)
\end{aligned}
$$

where $\rho_{m}=\sqrt{n} s_{m+1}$. Hence,

$$
\begin{aligned}
\frac{1}{v\left(B_{s}\right)} \sum_{k} v\left(M_{k} \cap B_{s}\right) \geq h_{m}( & -\frac{v\left(B_{s}\right)-v\left(B_{s-\rho_{m}}\right)}{v\left(B_{s}\right)} \\
& \left.-\frac{v\left(B_{t_{m}+\rho_{m}}\right)-v\left(B_{t_{m}-\rho_{m}}\right)}{v\left(B_{s}\right)}-\frac{v\left(B_{t_{m-1}+\rho_{m}}\right)}{v\left(B_{s}\right)}\right) .
\end{aligned}
$$

Since $t_{m}$ and $\rho_{m}$ have been chosen so that for $s \rightarrow \infty$ the terms with $v\left(B_{s}\right)$ in the denominator tend to 0 , and since $h_{m}$ tends to $d_{p}(\mathbf{K})$, we obtain (1). Using the fact that the definition of $d_{p}(\mathbf{K})$ shows that $\varlimsup_{s \rightarrow \infty}\left(1 / v\left(B_{s}\right)\right) \sum_{k} v\left(M_{k} \cap B_{s}\right) \leq d_{p}(\mathbf{K})$ we obtain that $\delta\left(\left\{M_{k}\right\}\right)$ exists and equals $d_{p}(\mathbf{K})$.

The proof for $d_{p}^{r}(K)$ is practically the same, except that it is not necessary (and not always possible) to avoid repeated use of the members of $K$. In other words, condition (iv) should be disregarded. 
In the case of coverings we proceed analogously but start with cubes of side length $s_{m}+4 b$ to obtain cubes $Q_{1}^{m}$ of side lengths $s_{m}$ so that (i) and (iv) are satisfied. Furthermore, instead of (ii) we can achieve that $Q_{i}^{m} \subset \bigcup_{j} L_{t, l}^{m}$, and instead of (iv) we can establish a similar relation with the inequality sign reversed and $h_{m}=\left(\left(s_{m}+4 b\right) / s_{m}\right)^{n}\left(d_{p}(\mathbf{K})+1 / m\right)$. Condition (iii) can be disregarded. Instead of (1) it has to be shown that $\varlimsup_{s \rightarrow \infty}\left(1 / v\left(B_{s}\right) \sum_{k} v\left(M_{k} \cap B_{s}\right) \leq d_{p}(\mathbf{K})\right.$. To prove this the necessary changes, as compared to the case of packings, are quite evident. Perhaps the only additional matter that deserves mentioning is that the term $\left(1 / v\left(B_{s}\right)\right) \sum_{k} v\left(M_{k} \cap B_{t_{m-1}}\right)$ cannot be disregarded. However, we have

$$
\begin{aligned}
\frac{1}{v\left(B_{s}\right)} \sum_{k} v\left(M_{k} \cap B_{t_{m-1}}\right) & \leq \frac{1}{v\left(B_{s}\right)} \sum_{Q_{i}^{k} \cap B_{t_{m-1}} \neq \varnothing} h_{k} v\left(Q_{1}^{k}\right) \\
& \leq \frac{h_{0}}{v\left(B_{s}\right)} v\left(B_{t_{m-1}+\rho_{m}}\right),
\end{aligned}
$$

and the definition of $t_{m}$ and $\rho_{m}$ shows that this term tends to 0 as $s \rightarrow \infty$.

Proof of the Continuity Theorem. First we need the following auxiliary statement: If $K$ is a convex body in $E^{n}$ that contains the ball $B_{a}$ and if $\varepsilon>0$, then

$$
K^{\varepsilon} \subset\left(1+\frac{\varepsilon}{a}\right) K
$$

where $K^{\varepsilon}$ denotes the parallel body of $K$ at distance $\varepsilon$. To prove this we introduce the support function, say $H(K, u)$, of $K$. Because of $B_{a} \subset K$ we have $H(K, u) \geq a$ and therefore $H\left(K^{\varepsilon}, u\right)=H(K, u)+\varepsilon \leq(1+\varepsilon / a) H(K, u)=H((1+\varepsilon / a) K, u)$.

We also make use of the following remark: If $\left\{X_{t}\right\}$ is a packing of convex bodies whose circumradii are at most $b$, and if each $X$, contains a $Y$, with $v\left(Y_{t}\right) \geq \lambda v\left(X_{t}\right)$, then $\bar{\delta}\left(\left\{Y_{1}\right\}\right) \geq \lambda \bar{\delta}\left(\left\{X_{1}\right\}\right)$. This follows immediately from

$$
\begin{aligned}
\bar{\delta}\left(\left\{Y_{t}\right\}\right) & =\varlimsup_{t \rightarrow \infty} \frac{1}{v\left(B_{t}\right)} \sum_{Y_{t} \cap B_{t} \neq \varnothing} v\left(Y_{t}\right) \\
& \geq \varlimsup_{t \rightarrow \infty} \frac{\lambda}{v\left(B_{t}\right)} \sum_{X_{i} \subset B_{t}} v\left(X_{i}\right)=\lambda \bar{\delta}\left(\left\{X_{i}\right\}\right) .
\end{aligned}
$$

Let now $\left\{P_{i}\right\}$ be a $\mathbf{K}$-packing and let $\mathbf{P}^{+}=\left\{P_{i}^{+}\right\}$be the corresponding expanded packing defined by $P_{i}^{+}=(1+\varepsilon / a) P_{i}$. Obviously, $\bar{\delta}\left(\left\{P_{i}^{+}\right\}\right)=\bar{\delta}\left(\left\{P_{i}\right\}\right)$. If $K_{\nu} \in \mathbf{K}, L_{\nu} \in \mathbf{L}$, and $h\left(K_{\nu}, L_{\nu}\right) \leq \varepsilon$, then, because of $(2), L_{\nu} \subset(1+\varepsilon / a) K_{\nu}+p_{\nu}$ and $K_{\nu} \subset(1+\varepsilon / a) L_{\nu}+q_{\nu}$, where $p_{\nu} \in E^{n}, q_{\nu} \in E^{n}$. Hence, there is a $r_{\nu} \in E^{n}$ so that $(1+\varepsilon / a)^{-1} K_{\nu}+r_{\nu} \subset L_{\nu}-p_{\nu} \subset(1+\varepsilon / a) K_{\nu}$. For each $i$ there are $\sigma_{1} \in G$ and a subscript $\nu_{i}$ so that $P_{i}^{+}=\sigma_{i}\left((1+\varepsilon / a) K_{\nu_{i}}\right)$. It follows that for some $x_{i} \in E^{n}$, $u, \in E^{n}$

$$
\left(1+\frac{\varepsilon}{a}\right)^{-1} \sigma_{i} K_{p_{i}}+x_{i} \subset \sigma_{i} L_{y_{i}}+u_{i} \subset P_{i}^{+}
$$


Setting $X_{i}=P_{i}^{+}, Y_{i}=(1+\varepsilon / a)^{-1} \sigma_{i} K_{v}+q_{i}$, and using the above remark we obtain $\bar{\delta}\left(\left\{Y_{t}\right\}\right) \geq(1+\varepsilon / a)^{-2 n} \bar{\delta}\left(\mathbf{P}^{+}\right)=(1+\varepsilon / a)^{-2 n} \bar{\delta}(\mathbf{P})$. Hence, if we define $M_{t}$ $=\sigma_{i} L_{v}+u_{i}$, then $\left\{M_{i}\right\}$ is an L-packing with $Y_{i} \subset M_{i-}$ and therefore $\bar{\delta}\left(\left\{M_{i}\right\}\right) \geq$ $(1+\varepsilon / a)^{-2 n} \delta(\mathbf{P})$. It follows that $d_{p}(\mathbf{L}) \geq(1+\varepsilon / a)^{-2 n} \bar{\delta}(\mathbf{P})$ and, since this is true for every $\mathbf{K}$-packing $\mathbf{P}$, we have

$$
\left(1+\frac{\varepsilon}{a}\right)^{2 n} d_{p}(\mathbf{L}) \geq d_{p}(\mathbf{K}) .
$$

Because of $\varepsilon / a<1$ we find

$$
d_{p}(\mathbf{K})-d_{p}(\mathbf{L}) \leq\left(\left(1+\frac{\varepsilon}{a}\right)^{2 n}-1\right) d_{p}(\mathbf{L}) \leq \frac{\varepsilon}{a}\left(4^{n}-1\right),
$$

and since $K$ and $\mathbf{L}$ can be interchanged we obtain the desired result. For $K^{r}$-packings the proof is essentially the same.

If $\mathbf{C}=\left\{C_{i}\right\}$ is a $\mathbf{K}$-covering we consider the contracted covering $C_{t}^{-}$defined by $C_{i}^{-}=(1+\varepsilon / a)^{-1} C_{i}$ and derive in analogy to (3)

$$
C_{i}^{-} \subset \sigma_{i} L_{\nu}+v_{i} \subset\left(1+\frac{\varepsilon}{a}\right) \sigma_{i} K_{\nu_{i}}+t_{i}
$$

where $\sigma_{i}$ is such that $(1+\varepsilon / a)^{-1} C_{i}=\sigma_{i} K_{\nu}$, and $v_{i} \in E^{n}, t_{i} \in E^{n}$. Setting $N_{i}=$ $\sigma_{i} L_{\nu}+v_{i}$ we find that $\left\{N_{i}\right\}$ is an $L$-covering and we derive as in the case of packings that $\underline{\delta}\left(\left\{N_{i}\right\}\right) \leq(1+\varepsilon / a)^{2 n} \delta(\mathrm{C})$. As before, this yields

$$
d_{c}(\mathbf{L}) \leq\left(1+\frac{\varepsilon}{a}\right)^{2 n} d_{c}(\mathbf{K})
$$

and we find

$$
\left|d_{c}(\mathbf{K})-d_{c}(\mathbf{L})\right| \leq\left(\left(1+\frac{\varepsilon}{a}\right)^{2 n}-1\right) \max \left\{d_{c}(\mathbf{K}), d_{c}(\mathbf{L})\right\}
$$

The Continuity Theorem for $\mathbf{K}$-coverings is an obvious consequence of this inequality. Essentially the same proof yields the corresponding result for $\mathbf{K}^{r}$ coverings.

Proof of the Transference Theorem. We first show that $d_{p}(\mathbf{K}) \geq d_{p}^{r}\left(\mathbf{K}^{\prime}\right)$. Let $\left\{M_{i}\right\}$ be a $\left(\mathbf{K}^{\prime}\right)^{r}$-packing of density $d_{p}^{r}\left(\mathbf{K}^{\prime}\right)$, and assume that an $\varepsilon>0$ is given. Since $\sigma_{i} M_{i} \in \mathbf{K}^{\prime}$ (for some $\left.\sigma_{i} \in G\right)$ there is a $K_{p_{t}} \in \mathbf{K}$ with $h\left(K_{v}, \sigma_{i} M_{i}\right) \leq \varepsilon$. Moreover, the sets $K_{\nu}$ can be selected so that no $K_{\nu_{1}}$ is used more than once. If we define $\mathbf{M}=\left\{\sigma_{i} M_{i}\right\}$ and $\hat{\mathbf{K}}=\left\{K_{\nu_{1}}\right\}$, then $\hat{\mathbf{K}} \subset \mathbf{K}$ and, by the Continuity Theorem,

$$
d_{p}(\mathbf{K}) \geq d_{p}(\hat{\mathbf{K}}) \geq d_{p}(\mathbf{M})-\left|d_{p}(\mathbf{M})-d_{p}(\hat{\mathbf{K}})\right| \geq d_{p}(\mathbf{M})-\frac{\varepsilon}{a}\left(4^{n}-1\right),
$$

where $a>0$. For $\varepsilon \rightarrow 0$ this shows that $d_{p}(\mathbf{K}) \geq d_{p}(\mathbf{M}) \geq d_{p}^{r}\left(\mathbf{K}^{\prime}\right)$.

Let us now prove that $d_{p}(\mathbf{K}) \leq d_{p}^{r}\left(\mathbf{K}^{\prime}\right)$. For this purpose let $\left\{P_{i}\right\}$ be a K-packing of density $d_{p}(\mathbf{K})$. Then, $P_{i}=\sigma_{i} K_{v_{i}}$ with $\sigma_{i} \in G, K_{v_{t}} \in \mathbf{K}$, and no $K_{\nu_{i}}$ 
used more than once. Let $\mathbf{L}$ be the set of limit elements of $\left\{K_{\nu}\right\}$. We may assume $\mathbf{L} \neq \varnothing$, and it is obvious that $\mathbf{L}$ is closed and bounded. Moreover, for any $\varepsilon>0$ there is an $m$ such that $\inf _{L \in \mathbf{L}} h\left(L, K_{\nu}\right)<\varepsilon$ whenever $i>m$. If this were not the case there would exist a convergent subsequence, say $\tilde{K}$, of $K_{\nu}$, such that $h\left(L, \tilde{K}_{j}\right) \geq \varepsilon$ for all $L \in \mathbf{L}$. But this is impossible since $\tilde{K}_{j}$ converges to some $L_{0} \in \mathbf{L}$ and therefore $\lim _{j \rightarrow \infty} h\left(L_{0}, \tilde{K}_{j}\right)=0$. Thus, for each $K_{\nu_{\text {}}}$ with $i>m$ there is an $L_{i} \in \mathbf{L} \subset \mathbf{K}^{\prime}$ such that $h\left(L_{i}, K_{v_{t}}\right)<\varepsilon$. Using the Continuity Theorem and setting $\mathbf{L}^{\prime}=\left\{L_{i}\right\}$ we can infer that

$$
\begin{aligned}
d_{p}^{r}\left(\mathbf{K}^{\prime}\right) & \geq d_{p}^{r}(\mathbf{L}) \geq d_{p}\left(\mathbf{L}^{\prime}\right) \geq d_{p}\left(\left\{K_{\nu_{1}}\right\}_{i>m}\right)-\left|d_{p}\left(\left\{K_{\nu_{i}}\right\}_{i>m}\right)-d_{p}\left(\mathbf{L}^{\prime}\right)\right| \\
& \geq d_{p}\left(\left\{P_{i}\right\}_{i>m}\right)-\frac{\varepsilon}{a}\left(4^{n}-1\right) .
\end{aligned}
$$

Letting $\varepsilon$ tend to 0 and noting that $d_{p}\left(\left\{P_{t}\right\}_{i>m}\right)=d_{p}\left(\left\{P_{i}\right\}\right)$ we can conclude that $d_{p}^{r}\left(\mathbf{K}^{\prime}\right) \geq d_{p}\left(\left\{\boldsymbol{P}_{i}\right\}_{i>m}\right)=d_{p}\left(\left\{P_{i}\right\}\right)=\delta\left(\left\{P_{i}\right\}\right)=d_{p}(\mathbf{K})$, which contains the desired result.

A proof for coverings is obtained from that for packings by the substitution of $d_{c}$ and $d_{c}^{r}$ for $d_{p}$ and $d_{p}^{r}$, and obvious reversions of inequality signs.

Finally, we note that all three theorems could be generalized. For example, convexity is clearly not essential for the validity of the Existence Theorem. Generalizations to multiple packings and coverings are also possible.

\section{References}

1. L. Danzer, Zerlegbarkeit endlichdimensionaler Räume in kongruente Simplices. Math.-Phys. Semesterber. 15 (1968), 87.

2. G. Fejes Tóth, New results in the theory of packing and covering. In Convexity and Its Applications, 318-359, Birkhäuser Verlag, Basel, 1983.

3. L. Fejes Tóth, Lagerungen in der Ebene, auf der Kugel und im Raum. 2. Aufl., Springer-Verlag, Berlin-Heidelberg-New York, 1972.

4. H. Groemer, Über Zerlegungen des Euklidischen Raumes. Math. Zeitschr. 79 (1962), 364-375.

5. H. Groemer, Existenzsätze für Lagerungen im Euklidischen Raum. Math. Zeitschr. 81 (1963), $260-278$.

6. H. Groemer, On a covering property of convex sets. Proc. Amer. Math. Soc. 59 (1976), 346-352.

7. H. Groemer, Covering and packing properties of bounded sequences of convex sets, Mathematika 29 (1982), 18-31.

8. H. Groemer, Coverings and packings by sequences of convex sets. In Discrete Geometry and Convexity, 262-278, New York Academy of Sciences, New York, 1985.

9. C. A. Rogers, Packing and Covering. Cambridge University Press, Cambridge, 1964.

Received May 31, 1985. 